\title{
Serum Cytokine Levels in Behçet's Disease
}

\author{
Zeynep Meltem Akkurt, ${ }^{1 *}$ Mehtap Bozkurt, ${ }^{2}$ Derya Uçmak, ${ }^{1}$ Hatice Yüksel, ${ }^{3}$ \\ Haydar Uçak, ${ }^{1}$ Bilal Sula, ${ }^{1}$ Zeynep Gürsel Özkurt, ${ }^{4}$ Mehmet Yildiz, ${ }^{2}$ Dicle Akdeniz, ${ }^{2}$ \\ and Mustafa Arica ${ }^{1}$ \\ ${ }^{1}$ Department of Dermatology, Dicle University Faculty of Medicine, Diyarbakır, Turkey \\ ${ }^{2}$ Department of Physical Therapy and Rehabilitation, Dicle University Faculty of Medicine, \\ Diyarbakır, Turkey \\ ${ }^{3}$ Department of Biochemistry, Dicle University Faculty of Medicine, Department of Biochemistry, \\ Diyarbakır, Turkey \\ ${ }^{4}$ Department of Ophthalmology, Dicle University Faculty of Medicine, Diyarbakır, Turkey
}

\begin{abstract}
Background: The aim of this study is to investigate and compare the serum levels of various cytokines in patients with Behçet's Disease and healthy controls. Methods: Thirty-five patients with Behçet's disease and 29 age and sex-matched healthy controls were included in the study. The patients were separated into groups with active and inactive disease. Serum IL-2, IL-6, IL-8, IL-10, IL-17A, and IFN- $\gamma$ levels were determined using the enzyme-linked immunosorbent assay method. Cytokine levels of the two patient groups and healthy controls were compared using SPSS 15.0. Results: Ten patients with active disease
\end{abstract}

Key words: Behçet's disease; interleukin-8 and 25 patients with inactive disease were present. Serum IL-8 levels of active BD patients were higher compared to inactive patients $(P=0.048)$ and healthy controls $(P=0.02)$. IL-8 levels were correlated with the duration of symptoms $(r=0.490, P=$ $0.003)$ and time passed since diagnosis ( $r=$ $0.579, P<0.001)$. Conclusion: Behçet's disease involves complex interactions of cells of the immune system, mainly $\mathrm{T}$ lymphocytes and neutrophils. Further studies on the cytokine profile in Behçet's disease will aid in elucidation of its pathogenesis. J. Clin. Lab. Anal. 29:317-320, 2015. 2014 Wiley Periodicals, Inc.
Behçet's disease (BD) is a chronic systemic inflammatory disorder, standing out from autoimmune and autoinflammatory diseases due to its unique properties. It is characterized by recurrent episodes of oral and genital ulcers, uveitis, and various systems involvement $(1,2)$. The onset of BD usually occurs in the third or fourth decade, and it is rare in children or patients over the age of 50 . Turkey has the highest prevalence of BD in the world, with up to 421 per 100,000 persons affected. Behçet's disease shows a male preponderance in Middle Eastern countries and the Mediterranean basin; however, women are more commonly affected in Japan and Korea (1). The most strongly associated genetic factor to BD, HLA-B51, accounts for less than $20 \%$ of the genetic risk, which shows that there remain other factors to be discovered $(3,4)$.

Cytokines are low-molecular weight polypeptides taking part in cell communication and are involved in an array of different immunologic mechanisms. They are im- portant mediators of immunologic and inflammatory reactions (5). Furthermore, the discovery of new T-cell subtypes and the frequent identification of novel cytokines has hastened research into the pathogenesis of BD. The aim of this study was to investigate and compare the serum levels of various cytokines in patients with BD and healthy controls. The study was carried out at the Rheumatology, Dermatology, and Ophtalmology outpatient clinics of the Dicle University Hospital from February 2013 to May 2013. Thirty-five patients with BD and 29 age and sexmatched healthy controls were included. All patients were

Grant sponsor: Dicle University Scientific Research Commission.

*Correspondence to: Zeynep Meltem Akkurt, Dicle Universitesi Tip Fak Dermatoloji AD, Diyarbakir, Turkey. E-mail: meltem@doctor.com

Received 17 March 2014; Accepted 2 April 2014

DOI $10.1002 /$ jcla. 21772

Published online in Wiley Online Library (wileyonlinelibrary.com). 
TABLE 1. Criteria Present at Sample Collection

\begin{tabular}{lcc}
\hline & Active BD & Inactive BD \\
\hline Oral ulcers & $80 \%$ & $48 \%$ \\
Genital ulcers & $40 \%$ & $8 \%$ \\
Eye involvement & $70 \%$ & $48 \%$ \\
Pathergy & $90 \%$ & $56 \%$ \\
Erythema nodosum & $10 \%$ & 0 \\
Pseudofolliculitis & $40 \%$ & $40 \%$ \\
Big vessel involvement & 0 & 0 \\
Arthritis & 0 & $4 \%$ \\
\hline
\end{tabular}

$\mathrm{BD}$, Behçet's disease.

screened according to the International Study Group for Behçet's Disease criteria (6) and the presence at the time of the study and history of relevant symptoms were recorded in detail. Pathergy tests of all patients were performed. Active disease was defined as the presence at sample collection of three International Study Group for Behçet's Disease criteria (6). Patients who had been diagnosed as BD in the past who had less than three active criteria at the time of the study were considered to have inactive disease. Erythrocyte sedimentation rate and C-reactive protein were not regarded as criteria for active disease. All patients were receiving at least one form of treatment at the time of collection of the samples. Treatment modalities were as follows: Colchicine $(12,18.8 \%)$, disease modifying antirheumatic drugs (azathioprine, cyclosporine, methotrexate), alone $(4,6.3 \%)$ or in combination with colchicine ( 15 , $23.4 \%$ ) or corticosteroids $(4,6.3 \%)$. Patients on treatment with IFN or anti-TNF treatments were not included in the study. Approval of the Ethics Committee of Dicle University Faculty of Medicine was obtained and all subjects gave written informed consent. Venous blood samples were obtained and immediately centrifuged. Serums were kept at $-80^{\circ} \mathrm{C}$ until laboratory testing. The serum interleukin (IL)-2, IL-6, IL-8, interferon (IFN)- $\gamma$ (Invitrogen, Camarillo, CA) and IL-10 (Invitrogen, Frederick, MD, CA) and IL-17A (Biosource Europe S. A. Belgium) levels were determined using the enzyme-linked immunosorbent assay method according to the manufacturer's protocols.

Statistical analyses were performed using SPSS 15.0. Student's $t$-test and and Mann-Whitney U test were used for the analysis of normally distributed and nonnormally distribued data, respectively. A $P$-value $<0.05$ was considered significant.

When grouped according to activity, there were ten patients with active BD (seven males and three females) and 25 patients with inactive BD (11 males and 14 females). The clinical manifestations present in active and inactive patients are shown in Table 1. The mean ages of the groups were $31.90 \pm 3.51$ years, $33.80 \pm 10.85$ years, and $29.97 \pm$ 12.43 years in active $\mathrm{BD}$, inactive $\mathrm{BD}$ and controls, re- spectively $(P>0.05)$. In active and inactive patients, mean duration of symptoms (8.40 years and 6.42 years) and mean time passed since diagnosis (6.56 years and 3.68 years) were statistically similar.

Serum IL-8 levels of active BD patients (2.04 \pm $0.33 \mathrm{pg} / \mathrm{ml})$ were higher compared to inactive patients $(1.81 \pm 0.28 \mathrm{pg} / \mathrm{ml})(P=0.048)$ and healthy controls $(1.82 \pm 0.20 \mathrm{pg} / \mathrm{ml})(P=0.02)$. IL-8 levels were correlated with the duration of symptoms $(r=0.490, P=0.003)$ and time passed since diagnosis $(r=0.579, P<0.001)$. Serum levels of IL-2, 6, 10, 17A, and IFN- $\gamma$ were similar in all study groups $(P>0.05)$. Evaluation for an association between individual clinical manifestations and serum levels revealed no significant difference with all the measured cytokines.

Higher levels of IL-8 were detected in patients with active BD in this study. IL-8, also known as neutrophil activating factor, is a major cytokine which strongly attracts and activates leukocytes. It is involved in the conversion of mononuclear to granulocytic infiltration and causes increased adhesion of peripheral blood leukocytes to endothelial cells, thus linking immune system activation with endothelial alterations in BD. It is mainly produced by macrophages, T cells, granulocytes, and endothelial cells. Small amounts can also be produced by fibroblasts, keratinocytes, hepatocytes, and chondrocytes $(4,7,8)$. Various studies have shown the levels of IL- 8 to be significantly elevated in active BD compared with inactive $\mathrm{BD}(7,9)$. Despite this, there are studies reporting no difference between IL-8 levels and active and inactive disease (10).

IL-8 has been shown to be associated with different individual clinical manifestations in $\mathrm{BD}$, that is, oral lesions, skin lesions, ocular manisfestations in one study (8). Such an association was not detected in the present study. However, the levels of IL-8 were correlated with duration of symptoms and time passed since diagnosis. This might be due to the longer duration of inflammation in these patients. Very high increases in IL-8 mRNA expression have been observed within lesions of $\mathrm{BD}$. This may reflect the important in situ recruitment of both polynuclear and mononuclear cells. Since IL-8 is a strong activator of neutrophil function, this finding may support the implication of polynuclear cell hyperfunction in the pathogenesis of BD lesions (11). Levels of IL-8 may be useful in following disease activity, or in predicting patients with more severe disease.

Other than IL-8, increased Th1-associated cytokines such as IFN- $\gamma$, IL-12, and TNF- $\alpha$, as well as IL-2 and IL-6 have been documented in BD patients and it is thought that the immune response in $\mathrm{BD}$ is skewed toward the Th1 path (11). However, treatment with IFN- $\alpha$ and anti-TNF agents have only partially prevented the progression of $\mathrm{BD}$ (12). This shows that, as in the case of allergy and other autoimmune disorders, only the 
Th1-Th2 paradigm cannot fully account for the full mechanism of the disease (13). The presence of a high Th17/Th1 ratio has been shown in BD, which indicates that Th17 cells, a recently identified T-cell population, may be the missing piece of the puzzle (14). IL17A is a powerful proinflammatory mediator secreted by Th17 cells (15).

Increased serum IL-17A levels have been shown in active BD patients, suggesting that Th17 cells and the IL-17 pathway has an important role in acute attacks of the disease $(16,17)$. Other studies have demonstrated increases in IL-23 and 27, also Th17 cytokines, rather than IL-17A (18).

IL-6 is a key cytokine in BD as it plays a role in differentiation of CD4+ T cells to Th17 cells $(15,19)$. Other cytokines thought to play a part in the pathogenesis of BD are IL-10, a potent suppressor of inflammatory cytokines, IL-12, a potent immunoregulatory cytokine, IL-18, a proinflammatory cytokine $(4,20)$.

The main limitation of this study was that all of the patients had been diagnosed in the past and were already receiving treatment. Medications, such as glucocorticosteroids and immunosuppressives reduce transcription of the proinflammatory cytokines (IL-8, TNF- $\alpha$, IL-1 etc.). Colchicine has been shown to significantly decrease levels of IL-6,8 and TNF- $\alpha$ in patients with mucocutaneous BD to a normal level when used with levamisole (21). Cyclosporine can significantly inhibit the intraocular inflammation of BD patients and the expression of IL-17 and IFN- $\gamma$ (22). IFN- $\alpha$ has been shown to inhibit IL-17 expression and increase IL-10 production by peripheral blood mononuclear cells and CD4+ T cells in vitro (23). Anti-TNF- $\alpha$ therapy suppresses effector $\mathrm{T}$ cell differentiation in BD patients with uveitis (24). Therefore, it is difficult to assess the true levels of cytokines in studies including patients taking such medications (7).

We did not find it appropriate to stop the use of medications for sample collection. Another limitation was that due to the setting of the study, the patient group was mostly composed of patients with mucocutaneous and/or ocular involvement, in whom systemic inflammation might have been less severe. Results of cytokine studies in BD should not be evaluated singly and should be viewed as a whole. Inconsistency of the results of various studies on serum cytokine levels in BD may be due to differences in patient characteristics, admission criteria, setting of the studies, and even ethnic differences.

In conclusion, BD is a disease involving complex interactions of cells of the immune system, mainly $\mathrm{T}$ lymphocytes and neutrophils. Various alterations in cytokine signaling seem to play a major role in its pathogenesis. Further research should focus on the immune interactions in BD.

\section{REFERENCES}

1. Cho SB, Cho S, Bang D. New insights in the clinical understanding of Behçet's disease. Yonsei Med J 2012;53:35-42.

2. Türkcü FM, Cingü AK, Yüksel H, Cinar Y, Akkurt M, Sahin M, Ozkurt Z, Sahin A, Caça I. Mean platelet volume in ocular Behçet's disease. Sci World J 2013;2013:215912.

3. Geri G, Terrier B, Rosenzwajg M, Wechsler B, Touzot M, Seilhean $\mathrm{D}$, et al. Critical role of IL-21 in modulating TH17 and regulatory T cells in Behçet disease. J Allergy Clin Immunol 2011;128:655-664.

4. Pineton de Chambrun M, Wechsler B, Geri G, Cacoub P, Saadoun D. New insights into the pathogenesis of Behçet's disease. Autoimmun Rev 2012;11:687-98.

5. Pekiner FN, Aytugar E, Demirel GY, Borahan MO. Interleukin-2, interleukin- 6 and $\mathrm{T}$ regulatory cells in peripheral blood of patients with Behçet's disease and recurrent aphthous ulcerations. J Oral Pathol Med 2012;41:73-79.

6. International Study Group for Behçet's Disease. Criteria for diagnosis of Behçet's disease. Lancet 1990;335:1078-1080.

7. Durmazlar SPK, Ulkar GB, Eskioglu F, Tatlican S, Mert A, Akgul A. Significance of serum interleukin-8 levels in patients with Behcet's disease: High levels may indicate vascular involvement. Int J Dermatol 2009;48:259-264.

8. Gür-Toy G, Lenk N, Yalcin B, Aksaray S, Alli N. Serum interleukin8 as a serologic marker of activity in Behçet's disease. Int J Dermatol 2005; 44:657-680.

9. Mege JL, Dilsen N, Sanguedolce V, Gul A, Bongrand P, Roux $\mathrm{H}$, et al. Overproduction of monocyte derived tumor necrosis factor alpha, interleukin (IL) 6, IL-8 and increased neutrophil superoxide generation in Behçet's disease. A comparative study with familial Mediterranean fever and healthy subjects. J Rheumatol 1993;20:1544-1549.

10. Sahin S, Akoğlu T, Direskeneli H, Sen LS, Lawrence R. Neutrophil adhesion to endothelial cells and factors affecting adhesion in patients with Behçet's disease. Ann Rheum Dis 1996;55: 128-133.

11. Ben Ahmed M, Houman H, Miled M, Dellagi K, Louzir H. Involvement of chemokines and Th1 cytokines in the pathogenesis of mucocutaneous lesions of Behçet's disease. Arthritis Rheum 2004; 50:2291-2295.

12. Chi W, Zhu X, Yang P, Liu X, Lin X, Zhou H, et al. Upregulated IL-23 and IL-17 in Behçet patients with active uveitis. Invest Ophthalmol Vis Sci 2008;49:3058-3064.

13. Direskeneli H, Fujita H, Akdis CA. Regulation of TH17 and regulatory T cells in patients with Behçet disease. J Allergy Clin Immunol 2011;128:665-666.

14. Kim J, Park JA, Lee EY, Lee YJ, Song YW, Lee EB. Imbalance of Th17 to Th1 cells in Behçet's disease. Clin Exp Rheumatol 2010;28:S16-19.

15. Kim ES, Kim SW, Moon CM, et al. Interactions between IL17A, IL23R, and STAT4 polymorphisms confer susceptibility to intestinal Behcet's disease in Korean population. Life Sci 2012;90:740746.

16. Ekinci NS, Alpsoy E, Karakas AA, Yilmaz SB, Yegin O. IL-17A has an important role in the acute attacks of Behçet's disease. J Invest Dermatol 2010;130:2136-2138.

17. Hamzaoui K, Bouali E, Ghorbel I, Khanfir M, Houman H, Hamzaoui A. Expression of Th-17 and ROR $\gamma \mathrm{t}$ mRNA in Behçet's Disease. Med Sci Monit 2011;17:CR227-CR234.

18. Jiang S, Liu X, Luo L, Qu B, Huang X, Lin Y, et al. Serum levels of Th17-related cytokines in Behcet disease patients after cataract surgery. Mol Vis 2011;17:1425-1430.

19. Bettelli E, Carrier Y, Gao W, et al. Reciprocal developmental pathways for the generation of pathogenic effector TH17 and regulatory T cells. Nature 2006;441:235-238. 


\section{Akkurt et al.}

20. Oztas MO, Onder M, Gurer MA, Bukan N, Sancak B. Serum interleukin 18 and tumour necrosis factor-alpha levels are increased in Behcet's disease. Clin Exp Dermatol 2005;30:61-63.

21. Sun A, Wang YP, Chia JS, Liu BY, Chiang CP. Treatment with levamisole and colchicine can result in a significant reduction of IL-6, IL-8 or TNF-alpha level in patients with mucocutaneous type of Behcet's disease. J Oral Pathol Med 2009;38: 401-405.

22. Chi W, Yang P, Zhu X, et al. Production of interleukin-17 in Be- hcet's disease is inhibited by cyclosporin A. Mol Vis 2010;16:880886.

23. Liu X, Yang P, Wang C, Li F, Kijlstra A. IFN-alpha blocks IL17 production by peripheral blood mononuclear cells in Behcet's disease. Rheumatology (Oxford) 2011;50:293-298.

24. Sugita S, Kawazoe Y, Imai A, Yamada Y, Horie S, Mochizuki M. Inhibition of Th17 differentiation by anti-TNF-alpha therapy in uveitis patients with Behçet's disease. Arthritis Res Ther 2012;14:R99. 